\title{
Extended Multiset-Table-Algebra Operations
}

\author{
Iryna Glushko \\ Nizhyn Gogol State University, Ukraine
}

\begin{abstract}
The paper is focused on some theoretical questions of the Database Theory. Multiset table algebra is considered. The notion of a table specified using the notion of a multiset (or bag). A signature of multiset table algebra is filled up with new operations such as inner and outer joins, semi-join and aggregate operations. A formal mathematical semantics of these operations is defined. The special element NULL is inserted in the universal domain for define of the outer join.
\end{abstract}

\section{Introduction}

The relational data model is nowadays in widespread use as in database scientific research so and in practice. In its formal definition, originally proposed by E. Codd, the relational model is based on sets of tuples, i.e. it does not allow duplicate tuples in a relation [1]. There are many applications the most peculiar feature of which is multiplicity and repeatability data. For example, these are sociological polls of different population groups, calculations on DNA and others. Commercial relational database systems are almost invariably based on multisets instead of sets. In other words, tables are in general allowed to include duplicate tuples.

For example, the data model of SQL is relational in nature, as well as the relevant operations. However, unlike relational algebra, the tables manipulated by SQL are not relations, but, rather, multisets. The reason for this peculiarity is twofold [2]. First, this is due to a practical reason: since SQL tables may be very large, duplicate elimination might become a bottleneck for the computation of the query result. Second, SQL extends the set of query operators by means of aggregate functions, whose operands are in general required to be multisets of values.

So, naturally there is a need to expand possibilities of relational databases due to use of multisets (or bags). This problem was also considered in [2-8]. However this question requires specification and extension because in the specified works the due attention isn't paid to operations of inner and outer joins, semijoin and aggregate operations of multiset table algebra.

\section{Multiset: Basic Definitions}

Let's introduce the formal definition of multisets in terms of monograph [5].

Definition 1. A multiset $\alpha$ with basis $U_{\alpha}$ is a function $\alpha: U_{\alpha} \rightarrow\{1,2, \ldots\}$, where $U_{\alpha}$ is an arbitrary set (in a classical Cantor's understanding).

Let's agree a multiset $\alpha$ with basis $U_{\alpha}=\left\{d_{1}, \ldots, d_{k}\right\}$ to write down as $\left\{d_{1}^{n_{1}}, \ldots, d_{k}^{n_{k}}\right\}$, where $n_{i}$ is a number of duplicate of the element $d_{i}$ in the multiset $\alpha$, so $n_{i}=\alpha\left(d_{i}\right), i=1, \ldots, k$.

Let $\alpha$ be a multiset with basis $U_{\alpha}=$ dom $\alpha$. Here dom $\alpha$ is the range of definition of multiset as a function.

Definition 2. A characteristic function of multiset $\alpha$ is a function $\chi_{\alpha}: D \rightarrow\{0,1,2, \ldots\}$, the values of which are specified by the following piecewise schema:

$$
\chi_{\alpha}(d)=\left\{\begin{array}{l}
\alpha(d) \text { if } d \in d o m \alpha, \\
0, \text { else; }
\end{array}\right.
$$

for all $d \in D$, where $D$ is the universe of elements of multiset bases.

Definition 3. An empty multiset $\varnothing_{m}$ is a multiset a characteristic function of which is a constant function, value of which is everywhere equal zero.

Definition 4. A rank of finite multiset $\alpha$ is a sum of duplicate elements of its basis $\|\alpha\|=\sum_{d \in d o m \alpha} \alpha(d)$; wherein $\left\|\varnothing_{m}\right\|=0$.

Let's introduce a binary relation inclusion over multisets.

Definition 5. Multiset $\beta$ is included in multiset

$$
\begin{aligned}
& \alpha \quad(\beta \preceq \alpha) \text {, if } \\
& \quad U_{\beta} \subseteq U_{\alpha} \& \forall d\left(d \in U_{\beta} \Rightarrow \beta(d) \leq \alpha(d)\right) .
\end{aligned}
$$

Directly from definition follows that this relation is a partial order.

The 1-multisets are the multisets whose range of values is an empty set or a single-element set $\{1\}$. These multisets are the analogues of ordinary sets.

Analogs of standard set-theoretic operations and operations, which are using peculiarities of multisets and, therefore cannot be useful for (abstract) sets, are defined in terms of characteristic functions in monograph [5]. There are operations of multiset union $\bigcup_{A l l}$, intersection $\bigcap_{A l l}$, difference $\backslash_{A l l}$, which 
build multisets of general view. The Cartesian product of multiset $\otimes$, the operation $\operatorname{Dist}(\alpha)$, which build 1-multiset, and analog of a full image for multisets are defined too.

\section{Multiset Table Algebra: Basic Definitions}

Among the two sets that are considered, $\boldsymbol{A}$ is the set of attributes and $\boldsymbol{D}$ is the universal domain.

Definition 6. An arbitrary (finite) set of attributes $R \subseteq A$ is called the scheme.

Definition 7. A tuple of the scheme $R$ is a nominal set on pair $R, D$. The projection of this nominal set for the first component is equal to $R$. In other words, a tuple of the scheme $R$ is a function $s: R \rightarrow D$.

The set of all tuples on scheme $R$ is designated as $S(R)$ and the set of all tuples is designated as $S=\bigcup_{R \subseteq A} S(R)$.

Definition 8. A table is a pair $\langle\psi, R\rangle$, where the first component $\psi$ is an arbitrary multiset basis of which $\Theta(\psi)$ is an arbitrary set (in particular infinite) of tuples of the scheme $R$ and other component $R$ is a scheme of the table.

Let $(\langle\psi, R\rangle)_{1}$ be the first component of the pair $\langle\psi, R\rangle$, i.e., is the set $\psi$. Thus, a certain scheme is ascribed to every table. The set of all table on scheme $R$ is designated as $\Psi(R)$ and the set of all table is designated as $\Psi=\bigcup_{R \subseteq A} \Psi(R)$.

The notation $\operatorname{Occ}(s, \psi)$ denotes the number of duplicate tuple $s$ in the multiset $\psi$. Let's agree a multiset to write down as $\left\{s_{1}^{n_{1}}, \ldots, s_{k}^{n_{k}}\right\}$, where $n_{i}=\operatorname{Occ}\left(s_{i}, \psi\right), i=1, \ldots, k$, and $\Theta(\psi)=\left\{s_{1}, \ldots, s_{k}\right\}$ is a basis of the multiset $\psi$.

Definition 9. The multiset table algebra is the algebra $\left\langle\Psi, \Omega_{P, \Xi}\right\rangle$, where $\Psi$ is the set of all tables, $\Omega_{P, \Xi}=\left\{\bigcup_{A l l}^{R}, \cap_{A l l}^{R}, \backslash_{A l l}^{R}, \sigma_{p, R}, \pi_{X, R}, \underset{R_{1}, R_{2}}{\otimes}, R t_{\xi, R}, \sim_{R}\right\} \quad$ is the signature, $p \in P, \xi \in \Xi, X, R, R_{1}, R_{2} \subseteq A, P, \Xi$ are the sets of parameters.

The operations of signature $\Omega_{P, \Xi}$ are defined in [6].

Theorem 1. Any expression over multiset table algebra can be replaced by equivalent to him expression which uses only operations of selection, join, projection, union, difference and renaming.

Proof. To prove this statement we will show that operations of intersection and active complement can be expressed through the operations noted in formulation of theorem. Indeed, the following equalities hold: 1) $\left\langle\psi_{1}, R\right\rangle \cap_{A l l}^{R}\left\langle\psi_{2}, R\right\rangle=$ $\left.\left\langle\psi_{1}, R\right\rangle \backslash_{\text {All }}^{R}\left(\left\langle\psi_{1}, R\right\rangle \backslash_{\text {All }}^{R}\left\langle\psi_{2}, R\right\rangle\right) ; \quad 2\right) \quad \sim_{R}(\langle\psi, R\rangle)=$ $=C(\langle\psi, R\rangle) \backslash_{A l l}^{R}\langle\psi, R\rangle, \quad$ where $\quad C(\langle\psi, R\rangle)=$ $=\pi_{\left\{A_{1}\right\}, R}(\langle\psi, R\rangle) \underset{\left\{A_{1}\right\},\left\{A_{2}\right\}}{\otimes} \cdots \underset{\left\{A_{1}, \ldots, A_{n-1}\right\},\left\{A_{n}\right\}}{\otimes} \pi_{\left\{A_{n}\right\}, R}(\langle\psi, R\rangle)$ and $R=\left\{A_{1}, \ldots, A_{n}\right\}$ is a scheme of the table $\langle\psi, R\rangle$ (see, for example, $[5,10]$ ).

\section{Extended Operations}

The extended operations include inner and outer joins, semijoin, aggregate operations. Let's consider these operations one at a time.

\subsection{Inner Joins}

There are four kinds of inner join operations Cartesian join, natural join, join using attributes $A_{1}, \ldots, A_{n}$ and join on predicate $p$. Let's define them.

Definition 10. The Cartesian Join of table on scheme $R_{1}$ and table on scheme $R_{2}$, moreover $R_{1} \cap R_{2}=\varnothing$, is a binary parametric operation $C j$ of the form

$$
\begin{gathered}
\underset{C j}{C j}: \Psi\left(R_{1}\right) \times \Psi\left(R_{2}\right) \rightarrow \Psi\left(R_{1} \cup R_{2}\right), \\
R_{1}, R_{2} \\
\left\langle\psi_{1}, R_{1}\right\rangle \underset{R_{1}, R_{2}}{C j}\left\langle\psi_{2}, R_{2}\right\rangle=\left\langle\psi^{\prime}, R_{1} \cup R_{2}\right\rangle,
\end{gathered}
$$

where $\left\langle\psi_{1}, R_{1}\right\rangle \in \Psi\left(R_{1}\right), \quad\left\langle\psi_{2}, R_{2}\right\rangle \in \Psi\left(R_{2}\right)$.

The basis of the multiset $\psi^{\prime}$ is defined by follow: $\Theta\left(\psi^{\prime}\right)=\left\{s \mid \exists s_{1} \exists s_{2}\left(s_{1} \in \Theta\left(\psi_{1}\right) \wedge s_{2} \in \Theta\left(\psi_{2}\right) \wedge\right.\right.$ $\left.\left.\wedge s=s_{1} \cup s_{2}\right)\right\}$. The number of duplicates is given by the following formula: $\operatorname{Occ}\left(s, \psi^{\prime}\right)=\operatorname{Occ}\left(s_{1}, \psi_{1}\right) \cdot \operatorname{Occ}\left(s_{2}, \psi_{2}\right), \quad$ where $s \in \Theta\left(\psi^{\prime}\right)$ and $s=s_{1} \bigcup s_{2}$.

Example 1. Consider the two tables $\left\langle\psi_{1}, R_{1}\right\rangle$ and $\left\langle\psi_{2}, R_{2}\right\rangle$ are shown in Table 1 and Table 2, respectively. Let's $R_{1}=\{A, B, C\} \quad$ and $R_{2}=\{D, E, F\}$.

Table 1. $\left\langle\psi_{1}, R\right\rangle$

\begin{tabular}{|c|c|c|}
\hline $\mathrm{A}$ & $\mathrm{B}$ & $\mathrm{C}$ \\
\hline$a$ & $a$ & $c$ \\
\hline$a$ & $a$ & $c$ \\
\hline$b$ & $b$ & $a$ \\
\hline
\end{tabular}

Table 2. $\left\langle\psi_{2}, R\right\rangle$

\begin{tabular}{|c|c|c|}
\hline $\mathrm{D}$ & $\mathrm{E}$ & $\mathrm{F}$ \\
\hline$a$ & $a$ & $a$ \\
\hline$a$ & $a$ & $c$ \\
\hline$a$ & $a$ & $c$ \\
\hline$a$ & $a$ & $c$ \\
\hline
\end{tabular}


If we decided to obtain a Cartesian join of these tables the result would be as shown in Table 3 .

This result can also be present as $\left\langle\psi_{1}, R_{1}\right\rangle \underset{R_{1}, R_{2}}{C j}\left\langle\psi_{2}, R_{2}\right\rangle=\{\{\langle A, a\rangle,\langle B, a\rangle,\langle C, c\rangle$,

$\{<D, a>,<E, a>,<F, a>\}^{2},\{<A, a>,<B, a>$,

$<C, c>,<D, a>,<E, a>,<F, c>\}^{6},\{<A, b>$,

$<B, b>,<C, a>,<D, a>,<E, a>,<F, a>\}^{1}$,

$\{\langle A, b\rangle,\langle B, b\rangle,\langle C, a\rangle,\langle D, a\rangle,\langle E, a\rangle$,

$\left.<F, c>\}^{3}, R_{1} \cup R_{2}\right\}^{1}$, where $R_{1} \cup R_{2}=\{A, B, C, D$, $E, F\}$.

Table 3. $\left\langle\psi_{1}, R_{1}\right\rangle \underset{R_{1}, R_{2}}{C j}\left\langle\psi_{2}, R_{2}\right\rangle$

\begin{tabular}{|c|c|c|c|c|c|}
\hline $\boldsymbol{A}$ & $\boldsymbol{B}$ & $\boldsymbol{C}$ & $\boldsymbol{D}$ & $\boldsymbol{E}$ & $\boldsymbol{F}$ \\
\hline$a$ & $a$ & $c$ & $a$ & $a$ & $a$ \\
\hline$a$ & $a$ & $c$ & $a$ & $a$ & $c$ \\
\hline$a$ & $a$ & $c$ & $a$ & $a$ & $c$ \\
\hline$a$ & $a$ & $c$ & $a$ & $a$ & $c$ \\
\hline$a$ & $a$ & $c$ & $a$ & $a$ & $a$ \\
\hline$a$ & $a$ & $c$ & $a$ & $a$ & $c$ \\
\hline$a$ & $a$ & $c$ & $a$ & $a$ & $c$ \\
\hline$a$ & $a$ & $c$ & $a$ & $a$ & $c$ \\
\hline$b$ & $b$ & $a$ & $a$ & $a$ & $a$ \\
\hline$b$ & $b$ & $a$ & $a$ & $a$ & $c$ \\
\hline$b$ & $b$ & $a$ & $a$ & $a$ & $c$ \\
\hline$b$ & $b$ & $a$ & $a$ & $a$ & $c$ \\
\hline
\end{tabular}

Definition 11. The Inner Natural Join of table on scheme $R_{1}$ and table on scheme $R_{2}$ is a binary parametric operation written as $\underset{R_{1}, R_{2}}{\otimes}$, whose value is the table on scheme $R_{1} \cup R_{2}$ consisting of all the unions of compatible tuples of input tables. Hence,

$$
\begin{gathered}
\underset{R_{1}, R_{2}}{\otimes}: \Psi\left(R_{1}\right) \times \Psi\left(R_{2}\right) \rightarrow \Psi\left(R_{1} \cup R_{2}\right), \\
\left\langle\psi_{1}, R_{1}\right\rangle \underset{R_{1}, R_{2}}{\otimes}\left\langle\psi_{2}, R_{2}\right\rangle=\left\langle\psi^{\prime}, R_{1} \cup R_{2}\right\rangle,
\end{gathered}
$$

where $\psi_{1} \in \Psi\left(R_{1}\right), \psi_{2} \in \Psi\left(R_{2}\right)$.

In other words, each tuple of $\psi_{1}$ is paired with each tuple of $\psi_{2}$, regardless of whether it is a duplicate or not. The basis of the multiset $\psi^{\prime}$ is defined by follow: $\Theta\left(\psi^{\prime}\right)=\left\{s \mid \exists s_{1} \exists s_{2}\left(s_{1} \in \Theta\left(\psi_{1}\right) \wedge\right.\right.$ $\left.\left.\wedge s_{2} \in \Theta\left(\psi_{2}\right) \wedge s_{1} \approx s_{2} \wedge s=s_{1} \cup s_{2}\right)\right\}$. The relation $\approx$ is a binary relation of compatibility of tuples $s_{1} \approx s_{2} \stackrel{\text { dif }}{\Leftrightarrow} s_{1}\left|R=s_{2}\right| R$ and $s_{i} \mid R$ is the restrictions of tuple $s_{i}$ on the scheme $R$ [5].

The number of duplicates is given by the following formula:

$$
\operatorname{Occ}\left(s_{1} \cup s_{2}, \psi^{\prime}\right)=\operatorname{Occ}\left(s_{1}, \psi_{1}\right) \cdot \operatorname{Occ}\left(s_{2}, \psi_{2}\right),
$$

\footnotetext{
${ }^{1}$ The top index specifies the count of the tuple in the table.
}

where $s^{\prime} \in \Theta\left(\psi^{\prime}\right)$ and $s^{\prime}=s_{1} \bigcup s_{2}$.

Example 2. Consider the two tables $\left\langle\psi_{1}{ }^{\prime}, R_{1}{ }^{\prime}\right\rangle$ and $\left\langle\psi_{2}{ }^{\prime}, R_{2}{ }^{\prime}\right\rangle$ are shown in Table 4 and Table 5, respectively. Let's $R_{1}{ }^{\prime}=\{A, B, C\} \quad$ and $R_{2}{ }^{\prime}=\{A, B, D\}$.

Table 4. $\left\langle\psi_{1}{ }^{\prime}, R_{1}{ }^{\prime}\right\rangle$

\begin{tabular}{|c|c|c|}
\hline $\mathrm{A}$ & $\mathrm{B}$ & $\mathrm{C}$ \\
\hline$a$ & $b$ & $c$ \\
\hline$a$ & $c$ & $a$ \\
\hline$a$ & $c$ & $a$ \\
\hline$b$ & $b$ & $b$ \\
\hline
\end{tabular}

Table 5. $\left\langle\psi_{2}{ }^{\prime}, R_{2}{ }^{\prime}\right\rangle$

\begin{tabular}{|c|c|c|}
\hline $\mathrm{A}$ & $\mathrm{B}$ & $\mathrm{D}$ \\
\hline$a$ & $b$ & $a$ \\
\hline$a$ & $c$ & $a$ \\
\hline$c$ & $b$ & $a$ \\
\hline$c$ & $b$ & $a$ \\
\hline
\end{tabular}

If we decided to obtain an Inner Natural Join of these tables the result can be present as

$$
\begin{gathered}
\left\langle\psi_{1}{ }^{\prime}, R_{1}{ }^{\prime}\right\rangle_{R_{1}{ }^{\prime}, R_{2}} \underset{ }{ }{ }^{\prime}\left\langle\psi_{2}{ }^{\prime}, R_{2}{ }^{\prime}\right\rangle= \\
=\left\{\{<A, a>,<B, b>,<C, c>,<D, a>\}^{1},\right. \\
\left.\{<A, a>,<B, c>,<C, a>,<D, a>\}^{2}, R_{1}{ }^{\prime} \bigcup R_{2}{ }^{\prime}\right\},
\end{gathered}
$$
where $R_{1}{ }^{\prime} \cup R_{2}{ }^{\prime}=\{A, B, C, D\}$.

Definition 12. The Inner Join using $A_{1}, \ldots, A_{n}$ of table on scheme $R_{1}$ and table on scheme $R_{2}$, moreover $R_{1} \cap R_{2}=\left\{A_{1}, \ldots, A_{n}\right\}, \quad$ is a binary parametric operation of the form

$$
\begin{array}{r}
\underset{A_{1}, \ldots, A_{n}, R_{1}, R_{2}}{\otimes}: \Psi\left(R_{1}\right) \times \Psi\left(R_{2}\right) \rightarrow \Psi\left(R_{1} \cup R_{2}\right), \\
\left\langle\psi_{1}, R_{1}\right\rangle \underset{A_{1}, \ldots, A_{n}, R_{1}, R_{2}}{\otimes}\left\langle\psi_{2}, R_{2}\right\rangle=\left\langle\psi^{\prime}, R_{1} \cup R_{2}\right\rangle,
\end{array}
$$

where $\left\langle\psi_{1}, R_{1}\right\rangle \in \Psi\left(R_{1}\right), \quad\left\langle\psi_{2}, R_{2}\right\rangle \in \Psi\left(R_{2}\right)$.

Moreover all $A_{1}, \ldots, A_{n}$ are pairwise different, $n \geq 1$, and $R_{1} \cap R_{2}=\left\{A_{1}, \ldots, A_{n}\right\}$. If input tables have also other general attributes which differ from $A_{1}, \ldots, A_{n}$, before join they needs to be renamed.

The basis of the multiset $\psi^{\prime}$ is defined by follow: $\Theta\left(\psi^{\prime}\right)=\left\{s \mid \exists s_{1} \exists s_{2}\left(s_{1} \in \Theta\left(\psi_{1}\right) \wedge s_{2} \in \Theta\left(\psi_{2}\right) \wedge\right.\right.$

$\left.\left.\wedge \wedge \wedge_{i=1}^{n} s_{1}\left(A_{i}\right)=s_{2}\left(A_{i}\right) \wedge s=s_{1} \cup s_{2}\right)\right\}$.

The number of duplicates is given by the following formula:

$$
\operatorname{Occ}\left(s, \psi^{\prime}\right)=\operatorname{Occ}\left(s_{1}, \psi_{1}\right) \cdot \operatorname{Occ}\left(s_{2}, \psi_{2}\right),
$$

where $s \in \Theta\left(\psi^{\prime}\right)$ and $s=s_{1} \cup s_{2}$.

Example 3. Consider the two tables $\left\langle\psi_{1}{ }^{\prime}, R_{1}{ }^{\prime}\right\rangle$ and $\left\langle\psi_{2}{ }^{\prime}, R_{2}{ }^{\prime}\right\rangle$ are shown in Table 4 and Table 5. Let's find an Inner Join using $A$ of these tables. Both 
input tables have another general attribute $B$. We must to rename it before using operation. We take the attribute $B$ of the table $\left\langle\psi_{2}{ }^{\prime}, R_{2}{ }^{\prime}\right\rangle$ and rename it to $E$. As result we will get the table $\left\langle\tilde{\psi}_{2}{ }^{\prime}, \tilde{R}_{2}{ }^{\prime}\right\rangle=\left\{\{<A, a>,<E, b>,<D, a>\}^{1},\{<A, a>\right.$, $\left.\left.<E, c>,\langle D, a>\}^{1},\{<A, c\rangle,<E, b\right\rangle,\langle D, a\rangle\right\}^{2}$, $\left.\tilde{R}^{\prime}\right\}$, where $\tilde{R}_{2}^{\prime}=\{A, E, D\}$.

Then the result of the Inner Join using $A$ can be present as

$$
\begin{gathered}
\left\langle\psi_{1}{ }^{\prime}, R_{1}{ }^{\prime}\right\rangle \underset{A, R_{1}{ }^{\prime}, R_{2}{ }^{\prime}}{\otimes}\left\langle\psi_{2}{ }^{\prime}, R_{2}{ }^{\prime}\right\rangle= \\
=\left\langle\psi_{1}{ }^{\prime}, R_{1}{ }^{\prime}\right\rangle \otimes_{A, R_{1}{ }^{\prime}, \tilde{R}_{2}{ }^{\prime}}\left\langle\tilde{\psi}_{2}{ }^{\prime}, \tilde{R}_{2}{ }^{\prime}\right\rangle= \\
=\left\{\{<A, a>,<B, b>,<C, c>,<E, b>,<D, a>\}^{1},\right. \\
\{<A, a>,<B, b>,<C, c>,<E, c>,<D, a>\}^{1}, \\
\{<A, a>,<B, c>,<C, a>,<E, b>,<D, a>\}^{2}, \\
\left.\{<A, a>,<B, c>,<C, a>,<E, c>,<D, a>\}^{2}, R\right\},
\end{gathered}
$$

where $R=R_{1}{ }^{\prime} \cup \tilde{R}_{2}{ }^{\prime}=\{A, B, C, E, D\}$.

Let $p: S \times S \stackrel{\sim}{\rightarrow}\{$ true, false $\}$ be a partial binary predicate on the set of all tuples $S$ such that $\forall s_{1} \forall s_{2}\left(\left\langle s_{1}, s_{2}\right\rangle \in \operatorname{dom} p \wedge p\left(s_{1}, s_{2}\right)=\right.$ true $\left.\Rightarrow s_{1} \approx s_{2}\right)$.

Definition 13. The Inner Join on predicate $p$ of table on scheme $R_{1}$ and table on scheme $R_{2}$ is a binary partial parametric operation of the form $\underset{p, R_{1}, R_{2}}{\otimes}: \Psi\left(R_{1}\right) \times \Psi\left(R_{2}\right) \stackrel{\sim}{\rightarrow} \Psi\left(R_{1} \cup R_{2}\right)$, $\left\langle\psi_{1}, R_{1}\right\rangle_{p, R_{1}, R_{2}}^{\otimes}\left\langle\psi_{2}, R_{2}\right\rangle=\left\langle\psi^{\prime}, R_{1} \cup R_{2}\right\rangle$.

The range of definition of this operation is dom $\underset{p, R_{1}, R_{2}}{\otimes}=\left\{\left\langle\left\langle\psi_{1}, R_{1}\right\rangle,\left\langle\psi_{2}, R_{2}\right\rangle\right\rangle \mid \Theta\left(\psi_{1}\right) \times\right.$

$\left.\Theta\left(\psi_{2}\right) \subseteq \operatorname{dom} p\right\}$. The basis of the multiset $\psi^{\prime}$ is defined by follow: $\Theta\left(\psi^{\prime}\right)=\left\{s \mid \exists s_{1} \exists s_{2}\left(s_{1} \in \Theta\left(\psi_{1}\right) \wedge\right.\right.$ $\wedge s_{2} \in \Theta\left(\psi_{2}\right) \wedge p\left(s_{1}, s_{2}\right) \simeq$ true $\left.\left.\wedge s=s_{1} \cup s_{2}\right)\right\}$ and $\simeq$ is a generalized equality (strong Kleene's equality) [9].

The number of duplicates is given by the following formula:

$\operatorname{Occ}\left(s_{1} \cup s_{2}, \psi^{\prime}\right)==\operatorname{Occ}\left(s_{1}, \psi_{1}\right) \cdot \operatorname{Occ}\left(s_{2}, \psi_{2}\right)$, where $s^{\prime} \in \Theta\left(\psi^{\prime}\right)$ and $s^{\prime}=s_{1} \cup s_{2}$.

Example 4. Consider the two tables $\left\langle\psi_{1}{ }^{1}, R_{1}{ }^{\prime}\right\rangle$ and $\left\langle\psi_{2}{ }^{\prime}, R_{2}{ }^{\prime}\right\rangle$ are shown in Table 4 and Table 5, respectively. Let's find an Inner Join on predicate $p$ of these tables and $p\left(s_{1}, s_{2}\right) \simeq$ true $\stackrel{\text { def }}{\Leftrightarrow} s_{1}(A)=a \wedge$ $\wedge s_{2}(A)=a \wedge s_{1}(B)=c \wedge s_{2}(B)=c$, where $s_{1} \in \Theta\left(\psi_{1}^{\prime}\right), s_{2} \in \Theta\left(\psi_{2}^{\prime}\right)$. The result can be present as

$$
\begin{gathered}
\left\langle\psi_{1}{ }^{\prime}, R_{1}{ }^{\prime}\right\rangle \underset{p, R_{1}{ }^{\prime}, R_{2}}{\otimes}\left\langle\psi_{2}{ }^{\prime}, R_{2}{ }^{\prime}\right\rangle= \\
\left\{\{<A, a>,<B, c>,<C, a>,<D, a>\}^{2}, R_{1}{ }^{\prime} \cup R_{2}{ }^{\prime}\right\},
\end{gathered}
$$

where $R_{1}{ }^{\prime} \cup R_{2}{ }^{\prime}=\{A, B, C, D\}$.

Let's note the following obvious fact. The join $\underset{R_{1}, R_{2}}{\otimes}$ is extension of another arbitrary inner join operation in the following sense:

$$
\begin{gathered}
\left\langle t_{1}, R_{1}\right\rangle \underset{R_{1}, R_{2}}{C j}\left\langle t_{2}, R_{2}\right\rangle=\left\langle t_{1}, R_{1}\right\rangle_{R_{1}, R_{2}}^{\otimes}\left\langle t_{2}, R_{2}\right\rangle, \\
\left\langle t_{1}, R_{1}\right\rangle \underset{A_{1}, \ldots, A_{n}, R_{1}, R_{2}}{\otimes}\left\langle t_{2}, R_{2}\right\rangle=\left\langle t_{1}, R_{1}\right\rangle_{R_{1}, R_{2}}^{\otimes}\left\langle t_{2}, R_{2}\right\rangle, \\
\left(\left\langle t_{1}, R_{1}\right\rangle_{p, R_{1}, R_{2}}^{\otimes}\left\langle t_{2}, R_{2}\right\rangle\right)_{1} \subseteq\left(\left\langle t_{1}, R_{1}\right\rangle_{R_{1}, R_{2}}^{\otimes}\left\langle t_{2}, R_{2}\right\rangle\right)_{1} .
\end{gathered}
$$

The values of these operations in the left parts of these two equalities and inclusion must be defined.

Definition 14.The Semijoin of table on scheme $R_{1}$ and table on scheme $R_{2}$ is a binary parametric operation written as $\ltimes_{R_{1}, R_{2}}$, whose value is the table on scheme $R_{1}$ containing tuples of the first table which are included in the inner natural join of input tables. Thus,

$$
\begin{aligned}
& \ltimes_{R_{1}, R_{2}}: \Psi\left(R_{1}\right) \times \Psi\left(R_{2}\right) \rightarrow \Psi\left(R_{1}\right), \\
& \left\langle\psi_{1}, R_{1}\right\rangle \ltimes_{R_{1}, R_{2}}\left\langle\psi_{2}, R_{2}\right\rangle=\left\langle\psi^{\prime}, R_{1}\right\rangle,
\end{aligned}
$$

where $\left\langle\psi_{1}, R_{1}\right\rangle \in \Psi\left(R_{1}\right), \quad\left\langle\psi_{2}, R_{2}\right\rangle \in \Psi\left(R_{2}\right)$. The basis of the multiset $\psi^{\prime}$ is defined by follow: $\Theta\left(\psi^{\prime}\right)=\left\{s_{1} \mid s_{1} \in \Theta\left(\psi_{1}\right) \wedge \exists s_{2}\left(s_{2} \in \Theta\left(\psi_{2}\right) \wedge s_{1} \approx s_{2}\right)\right\}$.

The number of duplicates is given by the following formula:

$$
\operatorname{Occ}\left(s, \psi^{\prime}\right)=\operatorname{Occ}\left(s, \psi_{1}\right),
$$

where $s \in \Theta\left(\psi^{\prime}\right)$.

Example 5. Consider the two tables $\left\langle\psi_{1}{ }^{\prime}, R_{1}{ }^{\prime}\right\rangle$ and $\left\langle\psi_{2}{ }^{\prime}, R_{2}{ }^{\prime}\right\rangle$ are shown in Table 4 and Table 5, respectively. If we decided to obtain a Semijoin of these tables the results can be present as

$$
\begin{aligned}
& \left\langle\psi_{1}{ }^{\prime}, R_{1}{ }^{\prime}\right\rangle \ltimes_{R_{1}{ }^{\prime}, R_{2}{ }^{\prime}}\left\langle\psi_{2}{ }^{\prime}, R_{2}{ }^{\prime}\right\rangle=\{\{\langle A, a\rangle,\langle B, b\rangle, \\
& \left.<C, a>\}^{1},\{\langle A, a\rangle,\langle B, c\rangle,\langle C, a\rangle\}^{2}, R_{1}{ }^{\prime}\right\}, \\
& \left\langle\psi_{2}{ }^{\prime}, R_{2}{ }^{\prime}\right\rangle \ltimes_{R_{2}{ }^{\prime}, R_{1}{ }^{\prime}}\left\langle\psi_{1}{ }^{\prime}, R_{1}{ }^{\prime}\right\rangle=\{\{\langle A, a\rangle,\langle B, b\rangle \text {, } \\
& \left.\left.<D, a>\}^{1},\{<A, a\rangle,\langle B, c\rangle,\langle D, a\rangle\right\}^{2}, R_{2}{ }^{\prime}\right\} \text {. }
\end{aligned}
$$

So semijoin is not commutative.

\subsection{Outer Join}

We can lose information when using the inner join operations because the tuples which are not compatible will not be represented in the output table. The outer join operations use when it is necessary to consider the tuples of input tables which didn't get to result of the inner join operations.

Let NULL be a special element of the universal domain $\boldsymbol{D}$. NULL used to denote absent values in the output table. Let $s_{R, N U L L}$ be a constant tuple on scheme $R$, i.e. $s_{R, N U L}: R \rightarrow\{N U L L\}$. 
There is one logical scheme for definition of the outer join operations [5].

Let $\varphi: \Psi\left(R_{1}\right) \times \Psi\left(R_{2}\right) \stackrel{\sim}{\rightarrow} \Psi\left(R_{1} \cup R_{2}\right)$ be some partial binary operation on the set of all tables and $\left(\varphi\left(\left\langle\psi_{1}, R_{1}\right\rangle,\left\langle\psi_{2}, R_{2}\right\rangle\right)\right)_{1} \preceq\left(\left\langle\psi_{1}, R_{1}\right\rangle_{R_{1}, R_{2}}^{\otimes}\left\langle\psi_{2}, R_{2}\right\rangle\right)_{1}$ for all tables $\left\langle\left\langle\psi_{1}, R_{1}\right\rangle,\left\langle\psi_{2}, R_{2}\right\rangle\right\rangle \in \operatorname{dom} \varphi$.

Let's notice that the operations $\underset{R_{1}, R_{2}}{C j}, \underset{R_{1}, R_{2}}{\otimes}$, $\underset{A_{1}, \ldots, A_{n}, R_{1}, R_{2}}{\otimes}, \underset{p, R_{1}, R_{2}}{\otimes}$ are such.

We fix two tables $\left\langle\psi_{1}, R_{1}\right\rangle,\left\langle\psi_{2}, R_{2}\right\rangle$ from range of definition of the operation $\varphi$.

Then the table $\left\langle\psi_{1}, R_{1}\right\rangle$ takes the following form $\left\langle\psi_{1}, R_{1}\right\rangle=\left\langle\psi_{1} \bigcap_{\varphi} \psi_{2}, R_{1}\right\rangle \bigcup_{A l l}^{R_{1}}\left\langle\psi_{1}-\psi_{\varphi}, R_{1}\right\rangle$.

Consider the table $\left\langle\psi_{1} \bigcap_{\varphi} \psi_{2}, R_{1}\right\rangle=\left\langle\psi^{\prime}, R_{1}\right\rangle$. The basis of the multiset $\psi^{\prime}$ is defined by follow: $\Theta\left(\psi^{\prime}\right)=\left\{s_{1} \mid s_{1} \in \Theta\left(\psi_{1}\right) \wedge \exists s_{2}\left(s_{2} \in \Theta\left(\psi_{2}\right) \wedge\right.\right.$ $\left.\left.\wedge s_{1} \cup s_{2} \in \Theta\left(\left(\varphi\left(\left\langle\psi_{1}, R_{1}\right\rangle,\left\langle\psi_{2}, R_{2}\right\rangle\right)\right)\right)\right)\right\}$. The number of duplicates is given by the following formula: $\operatorname{Occ}\left(s_{1}, \psi^{\prime}\right)=\operatorname{Occ}\left(s_{1}, \psi_{1}\right)$, where $s_{1} \in \Theta\left(\psi^{\prime}\right)$.

Consider the table $\left\langle\psi_{1}-\psi_{\varphi}, R_{1}\right\rangle=\left\langle\psi^{\prime \prime}, R_{1}\right\rangle$. The basis of the multiset $\psi^{\prime \prime}$ is defined by follow: $\Theta\left(\psi^{\prime \prime}\right)=\left\{s_{1} \mid s_{1} \in \Theta\left(\psi_{1}\right) \wedge \forall s_{2}\left(s_{2} \in \Theta\left(\psi_{2}\right) \Rightarrow\right.\right.$ $\left.\left.\Rightarrow s_{1} \cup s_{2} \notin \Theta\left(\left(\varphi\left(\left\langle\psi_{1}, R_{1}\right\rangle,\left\langle\psi_{2}, R_{2}\right\rangle\right)\right)\right)\right)\right\}$. The number of duplicates is given by the following formula: $\operatorname{Occ}\left(s_{1}, \psi^{\prime \prime}\right)=\operatorname{Occ}\left(s_{1}, \psi_{1}\right)$, where $s_{1} \in \Theta\left(\psi^{\prime \prime}\right)$.

In other words, the tuples of the table $\left\langle\psi_{1} \bigcap_{\varphi} \psi_{2}, R_{1}\right\rangle$ are used in formation of result of the join operation, and tuples of the table $\left\langle\psi_{1}-\psi_{\varphi}, R_{1}\right\rangle$ are not used.

We obtain a representation of the table $\left\langle\psi_{2}, R_{2}\right\rangle$ replacing the roles of the tables $\left\langle\psi_{1}, R_{1}\right\rangle$ and $\left\langle\psi_{2}, R_{2}\right\rangle$ in the presentation of the table $\left\langle\psi_{1}, R_{1}\right\rangle$.

Let's notice that if the operation $\varphi$ coincides with the operation $\underset{R_{1}, R_{2}}{\otimes}$ then the table $\left\langle\psi_{1} \bigcap_{\varphi} \psi_{2}, R_{1}\right\rangle$ is the semi-join of the tables $\left\langle\psi_{1}, R_{1}\right\rangle$ and $\left\langle\psi_{2}, R_{2}\right\rangle$, i.e. $\left\langle\psi_{1} \bigcap_{\varphi} \psi_{2}, R_{1}\right\rangle=\left\langle\psi_{1}, R_{1}\right\rangle \ltimes_{R_{1}, R_{2}}\left\langle\psi_{2}, R_{2}\right\rangle$.

There are four kinds of the outer joins operations which are induced of the inner join operation $\varphi$ : outer left join, outer right join, outer full join and union join. Let's define them.

Consider the following inner natural joins $\left\langle\psi_{1}-\psi_{\varphi}, R_{1}\right\rangle_{R_{1}, R_{2} \backslash R_{1}}^{\otimes}\left\langle\left\{s_{R_{2} \backslash R_{1}, N U L}^{1}\right\}, R_{2} \backslash R_{1}\right\rangle=\left\langle\psi^{\prime}, R_{1} \cup R_{2}\right\rangle$, where $\Theta\left(\psi^{\prime}\right)=\left\{s_{1} \bigcup s_{R_{2} \backslash R_{1}, N U L}^{1} \mid s_{1} \in \Theta\left(\psi_{1} \frac{-\psi_{2}}{\varphi}\right)\right\}$, $\operatorname{Occ}\left(s^{\prime}, \psi^{\prime}\right)=\operatorname{Occ}\left(s_{1}, \psi_{1}-\psi_{\varphi}\right), \quad s^{\prime} \in \Theta\left(\psi^{\prime}\right)$, $s^{\prime}=s_{1} \bigcup s_{R_{2} \backslash R_{1}, N U L L}^{1}$ and $\left\langle\psi_{2}-\psi_{\varphi}, R_{2}\right\rangle \underset{R_{2}, R_{1} \backslash R_{2}}{\otimes}\left\langle\left\{s_{R_{1} \backslash R_{2}, N U L L}^{1}\right\}, R_{1} \backslash R_{2}\right\rangle=\left\langle\psi^{\prime \prime}, R_{1} \cup R_{2}\right\rangle$, where $\Theta\left(\psi^{\prime \prime}\right)=\left\{s_{R_{1} \backslash R_{2}, N U L}^{1} \bigcup s_{2} \mid s_{2} \in \Theta\left(\psi_{2} \underset{\varphi}{-} \psi_{1}\right)\right\}$, $\operatorname{Occ}\left(s^{\prime \prime}, \psi^{\prime \prime}\right)=\operatorname{Occ}\left(s_{2}, \psi_{2}-\psi_{\varphi}\right), \quad s^{\prime \prime} \in \Theta\left(\psi^{\prime \prime}\right)$, $s^{\prime \prime}=s_{R_{1} \backslash R_{2}, N U L L}^{1} \cup s_{2}$.

Definition 15. The Outer Left Join operation is a partial binary operation of the form $\varphi_{l}: \Psi\left(R_{1}\right) \times \Psi\left(R_{2}\right) \stackrel{\sim}{\rightarrow} \Psi\left(R_{1} \cup R_{2}\right), \quad$ where $\operatorname{dom} \varphi_{l}=\operatorname{dom} \varphi \quad$ and $\quad \varphi_{l}\left(\left\langle\psi_{1}, R_{1}\right\rangle,\left\langle\psi_{2}, R_{2}\right\rangle\right)=$ $=\varphi\left(\left\langle\psi_{1}, R_{1}\right\rangle,\left\langle\psi_{2}, R_{2}\right\rangle\right) \bigcup_{A l l}^{R_{1} \cup R_{2}}\left\langle\psi_{1}-\psi_{\varphi}, R_{1}\right\rangle_{R_{1}, R_{2} \backslash R_{1}}^{\otimes}$ $\underset{R_{1}, R_{2} \backslash R_{1}}{\otimes}\left\langle\left\{s_{R_{2} \backslash R_{1}, N U L}^{1}\right\}, R_{2} \backslash R_{1}\right\rangle$.

Definition 16. The Outer Right Join operation is a partial binary operation of the form $\varphi_{r}: \Psi\left(R_{1}\right) \times \Psi\left(R_{2}\right) \stackrel{\sim}{\rightarrow} \Psi\left(R_{1} \cup R_{2}\right), \quad$ where $\operatorname{dom} \varphi_{r}=\operatorname{dom} \varphi \quad$ and $\quad \varphi_{r}\left(\left\langle\psi_{1}, R_{1}\right\rangle,\left\langle\psi_{2}, R_{2}\right\rangle\right)=$ $\varphi\left(\left\langle\psi_{1}, R_{1}\right\rangle,\left\langle\psi_{2}, R_{2}\right\rangle\right) \bigcup_{\text {All }}^{R_{1} \cup R_{2}}\left\langle\psi_{2}-\psi_{\varphi}, R_{2}\right\rangle \underset{R_{2}, R_{1} \backslash R_{2}}{\otimes}$ $\underset{R_{2}, R_{1} \backslash R_{2}}{\otimes}\left\langle\left\{s_{R_{1} \backslash R_{2}, N U L}^{1}\right\}, R_{1} \backslash R_{2}\right\rangle$.

Definition 17. The Outer Full Join operation is a partial binary operation of the form $\varphi_{f}: \Psi\left(R_{1}\right) \times \Psi\left(R_{2}\right) \stackrel{\sim}{\rightarrow} \Psi\left(R_{1} \cup R_{2}\right), \quad$ where $\operatorname{dom} \varphi_{f}=\operatorname{dom} \varphi \quad$ and $\quad \varphi_{f}\left(\left\langle\psi_{1}, R_{1}\right\rangle,\left\langle\psi_{2}, R_{2}\right\rangle\right)=$ $\varphi\left(\left\langle\psi_{1}, R_{1}\right\rangle,\left\langle\psi_{2}, R_{2}\right\rangle\right) \bigcup_{A l l}^{R_{1} \cup R_{2}}\left\langle\psi_{1}-\psi_{\varphi}, R_{1}\right\rangle \underset{R_{1}, R_{2} \backslash R_{1}}{\otimes}$ $\underset{R_{1}, R_{2} \backslash R_{1}}{\otimes}\left\langle\left\{s_{R_{2} \backslash R_{1}, N U L L}^{1}\right\}, R_{2} \backslash R_{1}\right\rangle \bigcup_{A l l}^{R_{1} \cup R_{2}}$ $\bigcup_{\text {All }}^{R_{1} \cup R_{2}}\left\langle\psi_{2}-\psi_{\varphi}, R_{2}\right\rangle_{R_{2}, R_{1} \backslash R_{2}}^{\otimes}\left\langle\left\{s_{R_{1} \backslash R_{2}, N U L L}^{1}\right\}, R_{1} \backslash R_{2}\right\rangle$.

Definition 18. The Outer Union Join operation is a partial binary operation of the form $\varphi_{\mathrm{V}}: \Psi\left(R_{1}\right) \times \Psi\left(R_{2}\right) \stackrel{\sim}{\rightarrow} \Psi\left(R_{1} \cup R_{2}\right), \quad$ where $\operatorname{dom} \varphi=\operatorname{dom} \varphi \quad$ and $\varphi\left(\left\langle\psi_{1}, R_{1}\right\rangle,\left\langle\psi_{2}, R_{2}\right\rangle\right)=$ $=\left\langle\psi_{1}-\psi_{\varphi}, R_{1}\right\rangle_{R_{1}, R_{2} \backslash R_{1}}^{\otimes}\left\langle\left\{s_{R_{2} \backslash R_{1}, N U L L}^{1}\right\}, R_{2} \backslash R_{1}\right\rangle \bigcup_{A l l}^{R_{1} \cup R_{2}}$ $\bigcup_{\text {All }}^{R_{1} \cup R_{2}}\left\langle\psi_{2}-\psi_{\varphi}, R_{2}\right\rangle \underset{R_{2}, R_{1} \backslash R_{2}}{\otimes}\left\langle\left\{s_{R_{1} \backslash R_{2}, N U L L}^{1}\right\}, R_{1} \backslash R_{2}\right\rangle$. 
Example 6. Consider the two tables $\left\langle\psi_{1}{ }^{\prime}, R_{1}{ }^{\prime}\right\rangle$ and $\left\langle\psi_{2}{ }^{\prime}, R_{2}{ }^{\prime}\right\rangle$ shown in Table 4 and Table 5. The results of Outer Join operations would be as follows:

$$
\begin{aligned}
& \varphi_{l}\left(\left\langle\psi_{1}{ }^{\prime}, R_{1}{ }^{\prime}\right\rangle,\left\langle\psi_{2}{ }^{\prime}, R_{2}{ }^{\prime}\right\rangle\right)=\{\{<A, a>,<B, b>,<C, c>, \\
& <D, a>\}^{1},\{<A, a>,<B, c>,<C, a>,<D, a>\}^{2}, \\
& \left.\{<A, b>,<B, b>,<C, b>,<D, N U L L>\}^{1}, R_{1}{ }^{\prime} \cup R_{2}{ }^{\prime}\right\} ; \\
& \varphi_{r}\left(\left\langle\psi_{1}{ }^{\prime}, R_{1}{ }^{\prime}\right\rangle,\left\langle\psi_{2}{ }^{\prime}, R_{2}{ }^{\prime}\right\rangle\right)=\{\{<A, a>,<B, b>,<C, c>, \\
& <D, a>\}^{1},\{<A, a>,<B, c>,<C, a>,<D, a>\}^{2}, \\
& \left.\{<A, c>,<B, b>,<C, N U L L>,<D, a>\}^{2}, R_{1}{ }^{\prime} \cup R_{2}{ }^{\prime}\right\} ; \\
& \varphi_{f}\left(\left\langle\psi_{1}{ }^{\prime}, R_{1}{ }^{\prime}\right\rangle,\left\langle\psi_{2}{ }^{\prime}, R_{2}{ }^{\prime}\right\rangle\right)=\{\{<A, a>,<B, b>,<C, c>, \\
& <D, a>\}^{1},\{<A, a>,<B, c>,<C, a>,<D, a>\}^{2}, \\
& \{<A, b>,<B, b>,<C, b>,<D, N U L L>\}^{1},\{<A, c>, \\
& \left.<B, b>,<C, N U L L>,<D, a>\}^{2}, R_{1}{ }^{\prime} \cup R_{2}{ }^{\prime}\right\} ; \\
& \varphi_{U}\left(\left\langle\psi_{1}{ }^{\prime}, R_{1}{ }^{\prime}\right\rangle,\left\langle\psi_{2}{ }^{\prime}, R_{2}{ }^{\prime}\right\rangle\right)=\{\{<A, b>,<B, b>,<C, b>, \\
& <D, N U L L>\}^{1},\{<A, c>,<B, b>,<C, N U L L>, \\
& \left.<D, a>\}^{2}, R_{1}{ }^{\prime} \cup R_{2}{ }^{\prime}\right\} .
\end{aligned}
$$

\subsection{Aggregate Operations}

The five types of aggregate operations discussed in this article are SUM, AVERAGE, MAXIMUM, MINIMUM, COUNT. The aggregate operations transform a finite table into a table with single tuple and single attribute.

Consider the table $\langle\psi, R\rangle \in \Psi(R)$, where $\psi$ is a finite multiset and $A \in R$. Let $\alpha_{A}$ be a multiset of column with attribute $A$ of table $\langle\psi, R\rangle$ which contains all elements including duplicates.

Then $\Theta\left(\alpha_{A}\right)=\{d \mid \exists s(s \in \Theta(\psi) \wedge\langle A, d\rangle \in s)\}=$ $=\left\{d \mid\{\langle A, d\rangle\} \in \Theta\left(\left(\pi_{\{A\}, R}(\langle\psi, R\rangle)\right)\right)\right\}$ is an analogue of active domain of the attribute $A$ [5]. The number of duplicates of element $d \in \Theta\left(\alpha_{A}\right)$ is given by the following formula: $\quad \alpha_{A}(d)=$ $=\operatorname{Occ}\left(\{\langle A, d\rangle\},\left(\pi_{\{A\}, R}(\langle\psi, R\rangle)\right)_{1}\right)=\sum_{\substack{s \in \Theta(\psi), s(A)=d}} \operatorname{Occ}(s, \psi)$.

Let $2_{m}^{D^{\prime}}=\left\{\alpha \mid \Theta\left(\alpha_{A}\right) \in 2^{D^{\prime}}\right.$ be a family of all multisets, bases of which are the finite subsets of the set $\boldsymbol{D}^{\prime}$. Here $\boldsymbol{D}^{\prime} \subseteq \boldsymbol{D}$ is a subset of the universal domain.

Let Num is a numerical subset of the universal domain $\boldsymbol{D}$ that is closed under addition. Extend the set Num by the special element NULL. We will not extend the operation of addition to the case where at least one of the arguments is NULL.

Let's define the aggregate operations. At first the five aggregate functions - count, sum, average, maximum, minimum - are defined on a finite multiset and then these functions are transferred to the tables.

Definition 19. The aggregate operation $\operatorname{Sum}_{A, R}$ by the attribute $A$ of the finite table on scheme $R$, $A \in R$, is a unary parametric operation of the form $\operatorname{Sum}_{A, R}: \Psi(R) \rightarrow \Psi(\{A\})$,

$\operatorname{Sum}_{A, R}(\langle\psi, R\rangle)=\left\langle\left\{\left\{\left\langle A, \operatorname{Sum}\left(\alpha_{A}\right)\right\rangle\right\}\right\},\{A\}\right\rangle^{2}, \quad$ where $\langle\psi, R\rangle \in \Psi(R)$. The $\operatorname{Sum}\left(\alpha_{A}\right)$ function is applied to a column with attribute $A$ in the table $\langle\psi, R\rangle$, the result obtained is the sum of every value occurrence in $\alpha_{A}$. In addition, NULL values don't undertake in attention and it is assumed that the column contains only data of numeric type.

Thus, Sum: $2_{m}^{\text {Num }} \rightarrow$ Num,

$$
\operatorname{Sum}\left(\alpha_{A}\right)=\left\{\begin{array}{l}
N U L L \text { if } \Theta\left(\alpha_{A}\right)=\varnothing ; \\
N U L L \text { if } \Theta\left(\alpha_{A}\right)=\{N U L L\} ; \\
\sum_{\left.d \in \Theta\left(\alpha_{A}\right) \backslash N U L U\right)} d \alpha_{A}(d) \text { if } \Theta\left(\alpha_{A}\right) \backslash\{N U L L\} \neq \varnothing .
\end{array}\right.
$$

So, we have $\operatorname{Sum}\left(\left\{N U L L^{n}\right\}\right)=N U L L$, $\operatorname{Sum}\left(\left\{d_{1}^{n_{1}}, \ldots, d_{k}^{n_{k}}\right\}\right)=\sum_{i=1}^{k} d_{i} n_{i} \quad$ if all elements $d_{i}$, $i=\overline{1, k}$, differ from $N U L L$.

In the case of the empty table $\left\langle\psi_{\varnothing}, R\right\rangle$ we have $\operatorname{Sum}_{A, R}\left(\left\langle\psi_{\varnothing}, R\right\rangle\right)=\left\langle\left\{\langle\{A, N U L L\rangle\}^{1}\right\},\{A\}\right\rangle, \quad$ here $\psi_{\varnothing}=\varnothing_{m}$.

Example 7. Let $\langle\psi, R\rangle$ be the Table 6. Then $\operatorname{Sum}_{A, R}(\langle\psi, R\rangle)=\left\langle\left\{\{\langle A, 8\rangle\}^{1}\right\},\{A\}\right\rangle$,

$\operatorname{Sum}_{B, R}(\langle\psi, R\rangle)=\left\langle\left\{\langle\langle B, 6\rangle\}^{1}\right\},\{B\}\right\rangle$,

$\operatorname{Sum}_{C, R}(\langle\psi, R\rangle)=\left\langle\left\{\{\langle C, 6\rangle\}^{1}\right\},\{C\}\right\rangle$.

Table 6. $\langle\psi, R\rangle$

\begin{tabular}{|l|l|l|}
\hline \multicolumn{1}{|c|}{$\mathrm{A}$} & $\mathrm{B}$ & \multicolumn{1}{c|}{$\mathrm{C}$} \\
\hline NULL & 0 & 3 \\
\hline 2 & 1 & 1 \\
\hline 2 & 1 & 1 \\
\hline 2 & 1 & 1 \\
\hline 2 & 3 & NULL \\
\hline
\end{tabular}

Let $\leq$ be a linear order on the universal domain D .

\footnotetext{
${ }^{2}$ The top index 1 specifies that the table include the tuple $\quad\left\{\left\langle A, \operatorname{Sum}\left(\alpha_{A}\right)\right\rangle\right\} \quad$ only once, i.e. $\left\{\left\{\left\langle A, \operatorname{Sum}\left(\alpha_{A}\right)\right\rangle\right\}^{1}\right\}$ is $\{1\}$-multiset.
} 
Definition 20. The aggregate operation $\operatorname{Min}_{A, R}$ by the attribute $A$ of the finite table on scheme $R$, $A \in R$, is a unary parametric operation of the form $\operatorname{Min}_{A, R}: \Psi(R) \rightarrow \Psi(\{A\})$,

$\operatorname{Min}_{A, R}(\langle\psi, R\rangle)=\left\langle\left\{\left\{\left\langle A, \operatorname{Min}\left(\alpha_{A}\right)\right\rangle\right\}^{1}\right\},\{A\}\right\rangle, \quad$ where $\langle\psi, R\rangle \in \Psi(R)$. The $\operatorname{Min}\left(\alpha_{A}\right)$ function is applied to a column with attribute $A$ in the table $\langle\psi, R\rangle$, the result obtained is the minimum value among values of $\alpha_{A}$. In addition, NULL values don't undertake in attention.

Thus, Min: $2_{m}^{D} \rightarrow \boldsymbol{D}$,

$\operatorname{Min}\left(\alpha_{A}\right)=\left\{\begin{array}{l}N U L L \text { if } \Theta\left(\alpha_{A}\right)=\varnothing ; \\ N U L L \text { if } \Theta\left(\alpha_{A}\right)=\{N U L L\} ; \\ \min \left\{d \mid d \in \Theta\left(\alpha_{A}\right) \backslash\{N U L L\}\right\} \text { if } \Theta\left(\alpha_{A}\right) \backslash\{N U L L\} \neq \varnothing .\end{array}\right.$

We have $\operatorname{Min}\left(\varnothing_{m}\right)=N U L L$,

$\operatorname{Min}\left(\left\{N U L L^{n}\right\}\right)=N U L L$,

$\operatorname{Min}\left(\left\{d_{1}^{n_{1}}, \ldots, d_{k}^{n_{k}}\right\}\right)=\min \left\{d_{1}, \ldots, d_{k}\right\}$ if all elements $d_{i}, i=\overline{1, k}$, differ from NULL .

In the case of the empty table $\left\langle\psi_{\varnothing}, R\right\rangle$ we have $\operatorname{Min}_{A, R}\left(\left\langle\psi_{\varnothing}, R\right\rangle\right)=\langle\{\langle\langle A, N U L L\rangle\}\},\{A\}\rangle, \quad$ here $\psi_{\varnothing}=\varnothing_{m}$.

Example 8. Let $\langle\psi, R\rangle$ be the Table 6. Then $\left.\operatorname{Min}_{A, R}(\langle\psi, R\rangle)=\left\langle\{\{\langle A, 2\rangle\}\}^{1}\right\},\{A\}\right\rangle$,

$\operatorname{Min}_{B, R}(\langle\psi, R\rangle)=\left\langle\left\{\{\langle B, 0\rangle\}^{1}\right\},\{B\}\right\rangle$,

$\operatorname{Min}_{C, R}(\langle\psi, R\rangle)=\langle\{\{\langle C, 1\rangle\}\},\{C\}\rangle$.

Definition 21. The aggregate operation $\operatorname{Max}_{A, R}$ by the attribute $A$ of the finite table on scheme $R$, $A \in R$, is a unary parametric operation of the form $\operatorname{Max}_{A, R}: \Psi(R) \rightarrow \Psi(\{A\})$,

$\operatorname{Max}_{A, R}(\langle\psi, R\rangle)=\left\langle\left\{\left\{\left\langle A, \operatorname{Max}\left(\alpha_{A}\right)\right\rangle\right\}\right\},\{A\}\right\rangle, \quad$ where $\langle\psi, R\rangle \in \Psi(R)$. The $\operatorname{Max}\left(\alpha_{A}\right)$ function is applied to a column with attribute $A$ in the table $\langle\psi, R\rangle$, the result obtained is the maximum value among values of $\alpha_{A}$. In addition, NULL values don't undertake in attention.

Thus, Max: $2_{m}^{D} \rightarrow \boldsymbol{D}$,

$\operatorname{Max}\left(\alpha_{A}\right)=\left\{\begin{array}{l}N U L L \text { if } \Theta\left(\alpha_{A}\right)=\varnothing ; \\ N U L L \text { if } \Theta\left(\alpha_{A}\right)=\{N U L L\} ; \\ \max \left\{d \mid d \in \Theta\left(\alpha_{A}\right) \backslash\{N U L L\}\right\} \text { if } \Theta\left(\alpha_{A}\right) \backslash\{N U L L\} \neq \varnothing .\end{array}\right.$

We have $\operatorname{Max}\left(\varnothing_{m}\right)=N U L L$,

$\operatorname{Max}\left(\left\{N U L L^{n}\right\}\right)=N U L L$,

$\operatorname{Max}\left(\left\{d_{1}^{n_{1}}, \ldots, d_{k}^{n_{k}}\right\}\right)=\max \left\{d_{1}, \ldots, d_{k}\right\}$ if all elements

$d_{i}, i=\overline{1, k}$, differ from NULL.
In the case of the empty table $\left\langle\psi_{\varnothing}, R\right\rangle$ we have $\operatorname{Max}_{A, R}\left(\left\langle\psi_{\varnothing}, R\right\rangle\right)=\langle\{\{\langle A, N U L L\rangle\}\},\{A\}\rangle, \quad$ here $\psi_{\varnothing}=\varnothing_{m}$.

Example 9. Let $\langle\psi, R\rangle$ be the Table 6. Then $\operatorname{Max}_{A, R}(\langle\psi, R\rangle)=\left\langle\left\{\left\{\langle A, 2\rangle^{1}\right\}\right\},\{A\}\right\rangle$,

$\operatorname{Max}_{B, R}(\langle\psi, R\rangle)=\left\langle\left\{\{\langle B, 3\rangle\}^{1}\right\},\{B\}\right\rangle$,

$\operatorname{Max}_{C, R}(\langle\psi, R\rangle)=\left\langle\left\{\{\langle C, 3\rangle\}^{1}\right\},\{C\}\right\rangle$.

Definition 22. The aggregate operation Count $_{A, R}$ by the attribute $A$ of the finite table on scheme $R$, $A \in R$, is a unary parametric operation of the form Count $_{A, R}: \Psi(R) \rightarrow \Psi(\{A\})$,

Count $_{A, R}(\langle\psi, R\rangle)=\left\langle\left\{\left\{\left\langle A, \operatorname{Count}\left(\alpha_{A}\right)\right\rangle\right\}^{1}\right\},\{A\}\right\rangle$, where $\langle\psi, R\rangle \in \Psi(R)$. The $\operatorname{Count}\left(\alpha_{A}\right)$ function is applied to a column with attribute $A$ in the table $\langle\psi, R\rangle$, the result obtained is the count of all values of $\alpha_{A}$ which differ from NULL.

Thus, Count $: 2_{m}^{D} \rightarrow\{0,1,2, \ldots\}$,

Count $\left(\alpha_{A}\right)=\sum_{\left.d \in \Theta\left(\alpha_{A}\right) \backslash N U L\right\}} \alpha_{A}(d)$. Put by definition that the sum of an empty set of elements is equal to zero.

So, we have $\operatorname{Count}\left(\varnothing_{m}\right)=0$,

$\operatorname{Count}\left(\left\{N U L L^{n}\right\}\right)=0$,

$\operatorname{Count}\left(\left\{d_{1}^{n_{1}}, \ldots, d_{k}^{n_{k}}\right\}\right)=n_{1}+\ldots+n_{k}$ if all elements $d_{i}$, $i=\overline{1, k}$, differ from $N U L L$.

In the case of the empty table $\left\langle\psi_{\varnothing}, R\right\rangle$ we have Count $_{A, R}\left(\left\langle\psi_{\varnothing}, R\right\rangle\right)=\left\langle\left\{\langle\langle A, 0\rangle\}^{1}\right\},\{A\}\right\rangle$, here $\psi_{\varnothing}=\varnothing_{m}$.

Example 10. Let $\langle\psi, R\rangle$ be the Table 6. Then Count $_{A, R}(\langle\psi, R\rangle)=\left\langle\left\{\langle\langle A, 4\rangle\}^{1}\right\},\{A\}\right\rangle$, Count $_{B, R}(\langle\psi, R\rangle)=\left\langle\left\{\langle\langle B, 5\rangle\}^{1}\right\},\{B\}\right\rangle$, Count $_{C, R}(\langle\psi, R\rangle)=\left\langle\left\{\langle\langle C, 4\rangle\}^{1}\right\},\{C\}\right\rangle$.

We assume that a numerical subset Num of the universal domain $\boldsymbol{D}$ is closed under the (partial operation) division operation /:Num $\times$ Num $\stackrel{\sim}{\rightarrow}$ Num. We will determine the division operation so that when the first argument is equal to $N U L L$ the function accepts value $N U L L$.

Definition 23. The aggregate operation $A v g_{A, R}$ by the attribute $A$ of the finite table on scheme $R$, $A \in R$, is a unary parametric operation of the form $A v g_{A, R}: \Psi(R) \rightarrow \Psi(\{A\})$,

$A v g_{A, R}(\langle\psi, R\rangle)=\left\langle\left\{\left\{\left\langle A, A v g\left(\alpha_{A}\right)\right\rangle\right\}\right\},\{A\}\right\rangle, \quad$ where 
$\langle\psi, R\rangle \in \Psi(R)$. The $\operatorname{Avg}\left(\alpha_{A}\right)$ function is applied to a column with attribute $A$ in the table $\langle\psi, R\rangle$, the result obtained is the arithmetic mean of values in $\alpha_{A}$ which differ from NULL. Thus, $\operatorname{Avg}: 2_{m}^{\text {Num }} \rightarrow \operatorname{Num}$ and $\operatorname{Avg}\left(\alpha_{A}\right)=\frac{\operatorname{Sum}\left(\alpha_{A}\right)}{\operatorname{Count}\left(\alpha_{A}\right)}$. We have $\operatorname{Avg}\left(\varnothing_{m}\right)=\frac{\operatorname{Sum}\left(\varnothing_{m}\right)}{\operatorname{Count}\left(\varnothing_{m}\right)}=\frac{N U L L}{0}=N U L L$, $\operatorname{Avg}\left(\left\{N U L L^{n}\right\}\right)=\frac{\operatorname{Sum}\left(\left\{N U L L^{n}\right\}\right)}{\operatorname{Count}\left(\left\{N U L L^{n}\right\}\right)}=N U L L$,

$\operatorname{Avg}\left(\left\{d_{1}^{n_{1}}, \ldots, d_{k}^{n_{k}}\right\}\right)=\frac{\operatorname{Sum}\left(\left\{d_{1}^{n_{1}}, \ldots, d_{k}^{n_{k}}\right\}\right)}{\operatorname{Count}\left(\left\{d_{1}^{n_{1}}, \ldots, d_{k}^{n_{k}}\right\}\right)}=$ $=\frac{\sum_{i=1}^{k} d_{i} n_{i}}{\left(n_{1}+\ldots+n_{k}\right)}$ if all elements $d_{i}, i=\overline{1, k}$, differ from $N U L L$.

In the case of the empty table $\left\langle\psi_{\varnothing}, R\right\rangle$ we have $A v g_{A, R}\left(\left\langle\psi_{\varnothing}, R\right\rangle\right)=\langle\{\{\langle A, N U L L\rangle\}\},\{A\}\rangle, \quad$ here $\psi_{\varnothing}=\varnothing_{m}$.

Example 11. Let $\langle\psi, R\rangle$ be the Table 6. Then $A v g_{A, R}(\langle\psi, R\rangle)=\langle\{\langle\{A, 2\rangle\}\},\{A\}\rangle$, $A v g_{B, R}(\langle\psi, R\rangle)=\left\langle\left\{\left\{\left\langle B, \frac{6}{5}\right\rangle\right\}^{1}\right\},\{B\}\right\rangle$ $A v g_{C, R}(\langle\psi, R\rangle)=\left\langle\left\{\left\{\left\langle C, \frac{6}{4}\right\rangle\right\}^{1}\right\},\{C\}\right\rangle$.

Definition 24. The aggregate operation Count $_{A, R}(*)$ by the attribute $A$ of the finite table on scheme $R, A \in R$, is a unary parametric operation of the form Count $_{A, R}(*): \Psi(R) \rightarrow \Psi(\{A\})$, Count $_{A, R}(*)(\langle\psi, R\rangle)=\left\langle\left\{\{\langle A,\|\psi\|\rangle\}^{t}\right\},\{A\}\right\rangle, \quad$ where $\langle\psi, R\rangle \in \Psi(R)$, and $\|\psi\|$ is the rank of the multiset $\psi$.

The operation $\operatorname{Count}_{A, R}(*)$ finds the number of tuples in the table $\langle\psi, R\rangle$.

In the case of an empty table $\left\langle\psi_{\varnothing}, R\right\rangle$ we have Count $_{A, R}(*)\left(\left\langle\psi_{\varnothing}, R\right\rangle\right)=\left\langle\left\{\left\{\left\langle A,\left\|\varnothing_{m}\right\|\right\rangle\right\}\right\},\{A\}\right\rangle=$ $=\langle\{\langle\langle A, 0\rangle\}\},\{A\}\rangle$, here $\psi_{\varnothing}=\varnothing_{m}$.

Example 12. Let $\langle\psi, R\rangle$ be the Table 6. Then Count $_{A, R}(*)(\langle\psi, R\rangle)=\left\langle\left\{\langle\langle A, 5\rangle\}^{1}\right\},\{A\}\right\rangle$,

$$
\begin{aligned}
& \text { Count }_{B, R}(*)(\langle\psi, R\rangle)=\left\langle\left\{\langle\langle B, 5\rangle\}^{1}\right\},\{B\}\right\rangle, \\
& \text { Count }_{C, R}(*)(\langle\psi, R\rangle)=\left\langle\left\{\langle\langle C, 5\rangle\}^{1}\right\}\{[C\}\rangle .\right.
\end{aligned}
$$

\section{Conclusions}

In this paper the multiset table algebra is considered. The concept of the table is specified, using concept of a multiset. The signature of the multiset table algebra is filled up with new operations such as inner and outer join, semijoin and aggregate operations. For each operations are defined a basis of the resulting table and number of duplicates of every tuple. The special element NULL is inserted in the universal domain for a define of outer operations.

It should also be noted that a parameter of aggregate operations is not necessarily only a single attribute; it also can be some function of the tuples.

\section{References}

[1] E.F. Codd, "A Relational Model for Large Shad Data Banks", Communications of the ACM, vo1.13, No.6, 1970, pp. 65-93.

[2] G. Lamperti, M. Melchiori, and M. Zanella, "On Multisets in Database Systems", Multiset Processing: Mathematical, Computer Science, and Molecular Computing Points of View, number 2235 in Lecture Notes in Computing Since, Berlin: Springer-Verlag, 2001, pp. 147-215.

[3] Paul W.P.J. Grefen, Rolf A. de By, "A Multi-Set Extended Relational Algebra. A Formal Approach to a Practical Issue", in Proceedings of the 10th International Conference on Data Engineering, ICDE, February 14-18, 1994, Houston, TX, USA, 1994, pp. 80-88.

[4] H. Garcia-Molina, J.D. Ullman, J. Widom, Database Systems:The Complete Book. Prentice Hall, Upper Saddle River, New Jersey, 2009.

[5] V. Redko, J. Brona, D. Buy, S. Poliakov, Relation Database: Relation Algebras and SQL-similar Languages. Kyiv, 2001. (in Ukrainian).

[6] D.B. Buy, I.M. Glushko, "Extended of Table Algebr: Multiset Table Algebra", Modern scientific research and their practical application, vol.J11309, Article CID Number 261, May, 2013.

[7] A. Silberschatz, H. Korth, S. Sudarshan, Database System Concepts. McGraw-Hill, 2011.

[8] I. Glushko "A Formal Mathematical Semantics of Advanced Operations of Multiset Table Algebra" in Proceedings of the 7th International Conference on Information Technology, Amman: Al-Zaytoonah University of Jordan, 2015, P. 369-375. 
International Journal of Intelligent Computing Research (IJICR), Volume 7, Issue 2, June 2016

[9] N. Cutland, Computability. An introduction to recursive function theory. Cambridge University Press, London. 1980.

[10] A.B. Petrovsky, Space of sets and multisets. Moscow, 2003. (in Russian) 